\title{
Management of adjuvant S-1 therapy after curative resection of gastric cancer: dose reduction and treatment schedule modification
}

\author{
Satoru Iwasa $\cdot$ Yasuhide Yamada $\cdot$ Takeo Fukagawa $\cdot$ \\ Takako Eguchi Nakajima $\cdot$ Ken Kato · Tetsuya Hamaguchi • \\ Shinji Morita $\cdot$ Makoto Saka $\cdot$ Hitoshi Katai $\cdot$ Yasuhiro Shimada
}

Received: 24 February 2010/ Accepted: 24 August 2010/Published online: 16 February 2011

(c) The International Gastric Cancer Association and The Japanese Gastric Cancer Association 2011

\begin{abstract}
Background The aim of this study was to determine the optimal management of adjuvant S-1 therapy for stage II or III gastric cancer, encompassing the details of dose reduction and treatment schedule modification.

Methods We retrospectively examined 97 patients with stage II or III gastric cancer who received S-1 chemotherapy following gastrectomy between January 2003 and December 2007. S-1 $\left(80 \mathrm{mg} / \mathrm{m}^{2}\right.$ per day) was orally administered twice daily for 4 weeks, followed by a 2-week rest. As a rule, treatment was continued for 1 year after gastrectomy. Dose reduction or treatment schedule modification was performed according to toxicity profiles. Results Among the 97 patients, 57 (59\%) underwent dose reduction at least once and $39(40 \%)$ received treatment schedule modification. Of the 57 patients who required dose reduction, $45(79 \%)$ underwent reduction within 3 months of the beginning of treatment. The most common reasons for dose reduction were anorexia (47\%), followed by diarrhea (32\%), leukopenia (24\%), and rash (16\%), with the reasons overlapping. Although the difference in the requirement for dose reduction was not significant, patients with a low creatinine clearance level or those who underwent total gastrectomy had a greater tendency to require dose reduction. The duration of the S-1 treatment period was at least 3 months in $88 \%$ of the patients, at least
\end{abstract}

S. Iwasa · Y. Yamada (凶) - T. Eguchi Nakajima - K. Kato ·

T. Hamaguchi · Y. Shimada

Gastrointestinal Oncology Division, National Cancer Center

Hospital, 5-1-1 Tsukiji, Chuo-ku, Tokyo 104-0045, Japan

e-mail: yayamada@ncc.go.jp

T. Fukagawa $\cdot$ S. Morita $\cdot$ M. Saka $\cdot$ H. Katai

Gastric Surgery Division, National Cancer Center Hospital,

Tokyo, Japan
6 months in $82 \%$, and the planned 1-year period in $73 \%$ of the patients.

Conclusions In most patients, the planned 1-year adjuvant S-1 therapy for stage II or III gastric cancer could be completed by modifying the dose reduction and treatment schedule.

Keywords S-1 · Adjuvant chemotherapy · Gastric cancer

\section{Introduction}

Gastric cancer remains one of the leading causes of cancerrelated deaths, the mortality rate of which ranks second worldwide [1]. Although surgery remains the sole mainstay of any curative treatment, the relapse rate is high and survival remains low even after surgical resection with curative intent. To prevent relapse and increase the survival rate, several types of adjuvant treatments have been administered. The rationale for using adjuvant treatment after curative resection remains controversial worldwide. In the United States, adjuvant chemoradiotherapy has become a standard treatment [2], and in Europe, perioperative chemotherapy has been established as a standard treatment [3]. On the other hand, in Japan, oral anticancer agents have been investigated for decades as postoperative adjuvant chemotherapy without sufficiently robust evidence for their efficacy. The different approaches in Europe, the United States, and Japan regarding adjuvant chemotherapy for gastric cancer may be attributable to differences in surgical approaches. In Europe and the United States, the standard surgical treatment is gastrectomy plus D0 or D1 lymphadenectomy, and chemoradiotherapy appears to be effective for local control after curative resection. In Japan, however, the established standard surgical treatment for gastric cancer is gastrectomy plus D2 
lymphadenectomy [4]. The role of adjuvant chemotherapy is to control distant or peritoneal recurrences. Against this background, adjuvant chemotherapy using oral fluorinated pyrimidines has been most widely used in both clinical practice and clinical trials [5, 6], although it remains unclear which specific treatment is effective.

$\mathrm{S}-1$ is an effective derivative of 5 -fluorouracil (5-FU) that combines tegafur with two modulators of 5-FU metabolism; namely, 5-chloro-2,4-dihydroxypyridine (CDHP), a reversible inhibitor of dihydropyrimidine dehydrogenase (DPD), and potassium oxonate, in a molar ratio of 1:0.4:1 [7]. Tegafur, an oral prodrug of 5-FU, is gradually converted to 5-FU and rapidly metabolized by DPD in the liver. The maximum concentration (Cmax) and area under the concentration time curve (AUC) of 5-FU in plasma during S-1 treatment have been found to be higher than the steady-state concentration and AUC of 5-FU in plasma during protracted intravenous infusion of 5 -FU at $250 \mathrm{mg} / \mathrm{m}^{2}$ per day [8]. Recently, the Adjuvant Chemotherapy Trial of TS- 1 for Gastric Cancer (ACTS-GC) was performed [9]. This was a large randomized controlled trial comparing surgery alone versus surgery plus adjuvant chemotherapy. Following the ACTS-GC trial, S-1 administration for 1 year after curative surgery increased both overall and relapse-free survival compared with surgery alone. Thus, S-1 has become widely used in Japan not only for unresectable recurrent or metastatic tumors but also for disease-free patients after curative surgery for gastric cancer.

In the ACTS-GC trial, even though the major grade $3 / 4$ toxicities included only anorexia $(6.0 \%)$, nausea $(3.7 \%)$, and diarrhea $(3.1 \%)$, the percentage of patients who completed the planned 1-year S-1 treatment was only $65.8 \%$. In addition, dose modification was performed in $42.4 \%$ of the patients, although details of the reasons for such modification were not provided. We therefore retrospectively investigated the details of dose reduction and schedule modification in patients with adjuvant S-1 therapy after curative resection of gastric cancer.

\section{Patients and methods}

Between January 2003 and December 2007, we retrospectively analyzed a total of 97 patients with stage II or III gastric cancer who received S-1 therapy after gastrectomy at the National Cancer Center Hospital, Tokyo. The clinicopathologic findings were determined in accordance with the Japanese classification of gastric carcinoma [10]. We regarded S-1 administration that started within 90 days after surgery as an acceptable period because this was a retrospective study, and because oncologists should consider starting adjuvant chemotherapy as soon as possible after gastrectomy (within 6 weeks) in order to eliminate micrometastases. There is no clear evidence for this 90 -day period, but it is approximately twice the starting limit of the ACTS-GC study. Moreover, because the period of adjuvant S-1 therapy was 1 year, this study was intended for patients who had started adjuvant S-1 therapy 1 year or more before the time of analysis. In principle, S-1 was administered orally at $40 \mathrm{mg}$ (body surface area $[\mathrm{BSA}]<1.25 \mathrm{~m}^{2}$ ), $50 \mathrm{mg}$ (BSA $1.25-1.50 \mathrm{~m}^{2}$ ), and $60 \mathrm{mg}\left(\right.$ BSA $>1.50 \mathrm{~m}^{2}$ ) twice daily for 4 weeks, followed by a 2 -week rest. However, a reduction of the starting dose was allowed at the physician's discretion; for example, when patients had postoperative gastrointestinal symptoms, poor general condition, or myelosuppression. S-1 administration was continued until 1 year after gastrectomy if there was no evidence of tumor recurrence or unacceptable toxicity. The dose or treatment schedule was modified at the physician's discretion according to toxicity profiles. In principle, we recommended that the schedule be changed from a 4-week administration followed by a 2 -week rest to a 2-week administration followed by a 1-week rest if patients could take S-1 twice per day completely without S-1 skip but had severe gastrointestinal symptoms or myelosuppression during the first 2 weeks, and the dose was reduced (1 level down) if sufficient S-1 could not be administered to the patients due to adverse events during first 2 weeks. Nevertheless, we recommended a further dose reduction of S-1 (2 levels down) or 2-week administration followed by a 1 -week rest when patients could not continue S-1 by adjusting first step dose or schedule modification. Furthermore, we attempted a 2-week administration followed by a 2-week rest or a 3-week administration followed by a 2-week rest if the treatment was not successful. We also allowed low doses such as $60 \mathrm{mg} /$ day when we could not manage adverse events with a reduction to $80 \mathrm{mg} /$ day.

The cumulative incidence of dose reduction was calculated by the Kaplan-Meier method, censoring at the date of treatment discontinuation caused by postoperative recurrence or adverse events. Statistical analysis was performed using Dr SPSS II (SPSS Japan, Tokyo, Japan). All statistical comparisons were two-sided and $P \leq 0.05$ was considered significant. Treatment-related toxicities were assessed using the Common Terminology Criteria for Adverse Events version 3.0.

This study was approved by the institutional review board of the National Cancer Center and was conducted in accordance with the ethical principles stated in Japanese ethics guidelines for epidemiological studies.

\section{Results}

Patient characteristics

The characteristics of the patients are shown in Table 1 . Forty patients underwent total gastrectomy, 54 had distal 
Table 1 Patient characteristics $(n=97)$

\begin{tabular}{ll}
\hline & $n(\%)$ \\
\hline Age (years) & \\
Median & 59 \\
Range & $35-80$ \\
Sex & \\
Male & $63(65)$ \\
Female & $34(35)$ \\
ECOG performance status & \\
0 & $60(62)$ \\
1 & $37(38)$ \\
Stage, Japanese classification & \\
II & $50(52)$ \\
IIIA & $29(30)$ \\
IIIB & $18(19)$ \\
Surgical procedure & \\
Total gastrectomy & $40(41)$ \\
Subtotal gastrectomy & $57(59)$ \\
Creatinine clearance (ml/min) & \\
$<60$ & $10(10)$ \\
$6-80$ & $29(30)$ \\
$>80$ & $58(60)$ \\
Initial dose of S-1 (mg/body) & \\
80 & $51(53)$ \\
100 & \\
120 & \\
\hline Creating & \\
& \\
& \\
&
\end{tabular}

Creatinine clearance was calculated using the Cockroft-Gault formula. Percentages might not add up to $100 \%$ due to rounding

ECOG Eastern Cooperative Oncology Group

gastrectomy, and the remaining 3 underwent pyloruspreserving gastrectomy. Of 19 patients receiving dose reduction at the initial administration, 6 showed inadequate food intake after gastrectomy, 5 had leucopenia and were judged unfit to start at the standard dose, 1 developed pancreatic fistula as a postsurgical complication, and 1 had borderline BSA (This patient had BSA of 1.51, so physician selected S-1 $100 \mathrm{mg} /$ day in consideration of postsurgical condition); the reasons for the dose reduction in the remaining 6 patients were unknown. Of the 97 patients enrolled, 62 patients exceeded the 6-week starting time limit of the ACTS-GC study. The reasons were pancreatic fistula in 6 patients, gastrointestinal symptoms in 5 patients, poor oral intake in 3 patients, poor general condition in 3 patients, another treatment in 2 patients (dental therapy in 1 and treatment for early bladder cancer in the other), anastomotic stenosis in 1 patient, ileus in 1 patient, colitis in 1 patient, delay of pathological confirmation in 1 patient, and by patient request for 1 patient; and the remaining 38 patients delayed seeking an examination at the outpatient clinic by about 1 or 2 weeks.
Table 2 Adverse events $(n=97)$

\begin{tabular}{|c|c|c|c|c|c|c|}
\hline & \multicolumn{4}{|c|}{ No. of patients } & \multirow{2}{*}{$\begin{array}{l}\% \\
\text { All G }\end{array}$} & \multirow{2}{*}{$\begin{array}{l}\% \\
\text { G3/4 }\end{array}$} \\
\hline & G1 & $\mathrm{G} 2$ & G3 & G4 & & \\
\hline \multicolumn{7}{|l|}{ Hematological toxicity } \\
\hline Leukopenia & 35 & 35 & 1 & 0 & 73 & 1 \\
\hline Neutropenia & 18 & 36 & 22 & 1 & 79 & 24 \\
\hline Anemia & 66 & 20 & 1 & 0 & 90 & 1 \\
\hline Thrombocytopenia & 18 & 2 & 0 & 0 & 21 & 0 \\
\hline \multicolumn{7}{|c|}{ Nonhematological toxicity } \\
\hline Anorexia & 67 & 11 & 3 & 0 & 84 & 3 \\
\hline Nausea & 43 & 5 & 0 & 0 & 49 & 0 \\
\hline Vomiting & 14 & 2 & 0 & 0 & 17 & 0 \\
\hline Mucositis & 35 & 4 & 0 & 0 & 40 & 0 \\
\hline Diarrhea & 53 & 10 & 2 & 0 & 67 & 2 \\
\hline Fatigue & 65 & 4 & 1 & 0 & 72 & 1 \\
\hline Rash & 27 & 3 & 1 & 0 & 32 & 1 \\
\hline Pigmentation & 40 & 4 & - & - & 45 & - \\
\hline Hand-foot syndrome & 11 & 2 & 1 & - & 14 & 1 \\
\hline Watery eyes & 6 & 5 & 1 & - & 12 & 1 \\
\hline Taste alteration & 13 & 5 & - & - & 19 & - \\
\hline Hypoalbuminemia & 23 & 2 & 1 & 0 & 27 & 1 \\
\hline Hyperbilirubinemia & 33 & 10 & 1 & 0 & 45 & 1 \\
\hline AST & 27 & 3 & 2 & 0 & 33 & 2 \\
\hline ALT & 22 & 2 & 2 & 0 & 27 & 2 \\
\hline ALP & 16 & 0 & 0 & 0 & 17 & 0 \\
\hline Creatinine & 6 & 1 & 0 & 0 & 7 & 0 \\
\hline
\end{tabular}

$G$ grade, $A S T$ aspartate aminotransferase, $A L T$ alanine aminotransferase, $A L P$ alkaline phosphatase

\section{Safety}

S-1 chemotherapy adverse events, which involved grade $1 / 2$ intensity in the majority of cases, are summarized in Table 2. The most common hematological adverse event was neutropenia, occurring at grade 3/4 intensity in 23 patients $(24 \%)$. Only 1 patient developed grade 3 febrile neutropenia. Nonhematological toxicity was frequent but rarely severe. Taste alteration and watery eyes, which occurred consistently with repeated courses of therapy, were experienced by $18(19 \%)$ and $12(12 \%)$ patients, respectively, and occasionally a few patients needed dose reduction or treatment discontinuation. There were no treatment-related deaths.

Treatment administration

The median follow-up period after gastrectomy was 43.0 months (range 5.3-73.4 months). Among the 97 patients analyzed, $57(59 \%)$ received dose reduction at least once and $39(40 \%)$ underwent schedule modification during the planned 1-year treatment. Of the 57 patients 


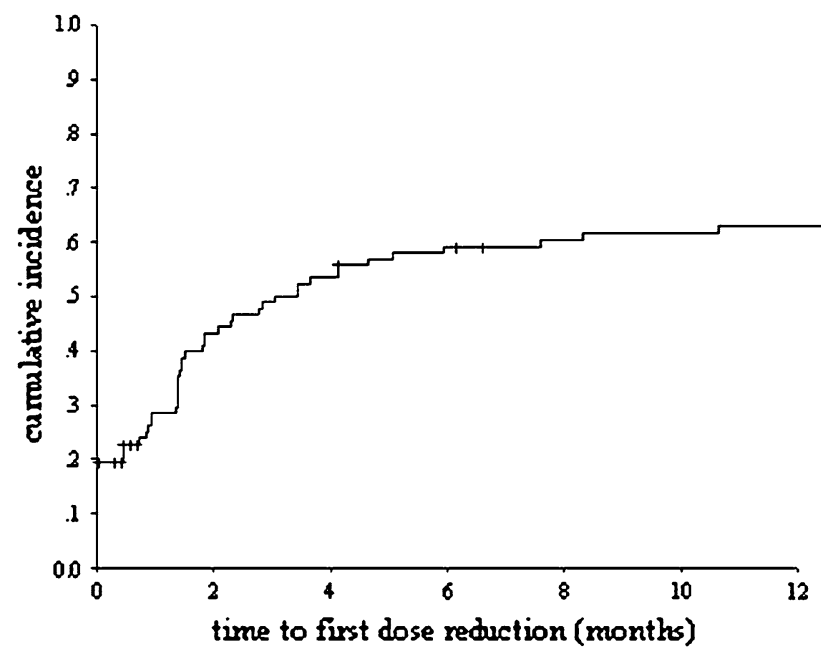

Fig. 1 Cumulative incidence of the time to first dose reduction

Table 3 Reasons for dose reduction $(n=57)$

\begin{tabular}{ll}
\hline Pretreatment dose reduction & $19(33 \%)$ \\
Inadequate food intake & 6 \\
Leukopenia & 5 \\
Pancreatic fistula & 1 \\
Borderline BSA* & 1 \\
Unknown & 6 \\
Dose reduction during the treatment & $38(67 \%)$ \\
Gastrointestinal toxicities & \\
Anorexia & 18 \\
Diarrhea & 12 \\
Nausea/vomiting & 5 \\
Mucositis & 2 \\
Abdominal pain & 1 \\
Others & \\
Leukopenia/neutropenia & 9 \\
Rash & 6 \\
Fatigue & 3 \\
Taste alteration & 3 \\
Liver dysfunction & 2 \\
Watery eyes & 1 \\
\hline
\end{tabular}

The percentages and numbers do not balance because of overlapping reasons for dose reduction during the treatment

$B S A$ body surface area

* 1 patient with borderline BSA had BSA of 1.51 , so physician selected S-1 $100 \mathrm{mg} /$ day in consideration of postsurgical condition

who required dose reduction, $45(79 \%)$ underwent the reduction within 3 months of starting the treatment. The median time to the first dose reduction was 1.4 months (range $0-10.6$ months). The time to dose reduction is shown graphically in Fig. 1. The most common reasons for dose reduction during the treatment period were anorexia
Table 4 Reasons for treatment discontinuation $(n=26)$

\begin{tabular}{ll}
\hline Adverse events & $20(77 \%)$ \\
Gastrointestinal toxicities & 14 \\
Anorexia & 6 \\
Diarrhea & 3 \\
Nausea/vomiting & 3 \\
Abdominal pain & 1 \\
Mucositis & 1 \\
Others & \\
Fatigue & 3 \\
Leukopenia/neutropenia & 2 \\
Watery eyes & 2 \\
Taste alteration & 2 \\
Rash & 1 \\
Hand-foot syndrome & 1 \\
Pigmentation & 1 \\
Liver function & 1 \\
Dyspnea & 1 \\
Recurrence & $2(8 \%)$ \\
Others & $4(15 \%)$ \\
\hline
\end{tabular}

The numbers do not balance because of overlapping reasons for treatment discontinuation

(47\%), followed by diarrhea (32\%), leukopenia (24\%), and rash (16\%) with reasons overlapping (Table 3). Of the 39 patients who required schedule modification, 22 patients underwent a 2-week administration followed by a 1-week rest, 19 patients had a 2-week administration followed by a 2 -week rest, and 2 patients had a 3 -week administration followed by a 2 -week rest (including the 4 patients who underwent two modifications, i.e., a 2 -week administration followed by a 1-week rest, and a 2-week administration followed by a 2 -week rest in 3 patients, and a 3 -week administration followed by a 2-week rest and a 2-week administration followed by a 2 -week rest in 1 patient).

A total of 60 patients required dose reduction and/or schedule modification, and 32 patients underwent remodification after their initial modification (the number of modifications was 1 in 28 patients, 2 in 22 patients, 3 in 9 patients, and 4 in 1 patient). No patients were rechallenged with the initial dose after undergoing dose reduction due to adverse events during the adjuvant chemotherapy. The relative administration day, defined as the ratio of actual administration days to planned administration days, was $83.8 \%$, and we regarded relative number of administration days assuming that was reckoned from the start date of actual S-1 administration, not within 6 weeks after surgery specified in ACTS-GC. The relative dose intensity, defined as the ratio of the actual cumulative dose to the planned cumulative dose, was $69.1 \%$. The percentage of patients who continued treatment for at least 3 months was $88 \%$, that of patients who continued treatment for at least 6 months was $82 \%$, and that 

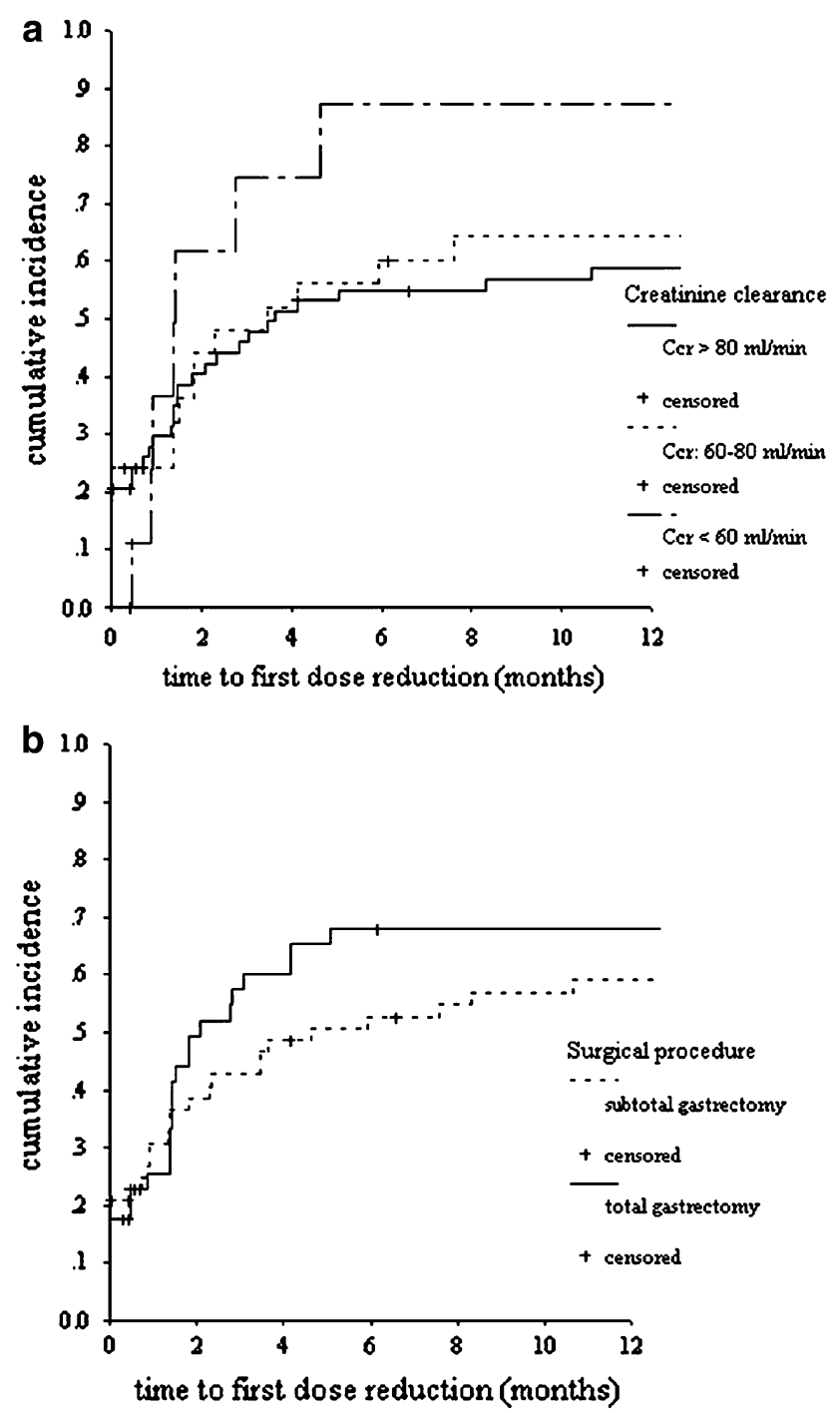

Fig. 2 Cumulative incidence of the first dose reduction. a By pretreatment creatinine clearance (Ccr) level; Ccr $>80 \mathrm{ml} / \mathrm{min}$ $(n=58)$, Ccr $60-80 \mathrm{ml} / \mathrm{min}(n=29)$, Ccr $<60 \mathrm{ml} / \mathrm{min}(n=10)$. Patients with a lower Ccr level had a greater tendency to require dose reduction $(P=0.20)$. b By surgical procedures; subtotal gastrectomy group $(n=57)$, total gastrectomy group $(n=40)$. Patients with total gastrectomy had a greater tendency to require dose reduction $(P=0.36)$

of patients who continued treatment for the scheduled 12 months was $73 \%$. Twenty-six patients (27\%) discontinued treatment (Table 4). The reasons for treatment discontinuation were adverse events in 20 patients, recurrent disease in 2 , and other reasons in 4 . The median duration until treatment discontinuation was 4.0 months.

We performed univariate analyses using pretreatment patient profiles (sex, age, performance status, surgical procedure, creatinine clearance, and time interval between surgery and first S-1 administration) as the basis of dose reduction during the planned 1-year treatment. Although the univariate analyses revealed no significant predictive factors for dose reduction, the incidence of dose reduction tended to be higher in patients with a low creatinine clearance level and in those who underwent total gastrectomy (Fig. 2). Moreover, no significant difference in recurrence was found depending on the $\mathrm{S}-1$ starting time (within 6 weeks vs. more than 6 weeks; data not shown).

\section{Discussion}

The present study demonstrated that in most patients, the planned 1-year adjuvant S-1 therapy for stage II or III gastric cancer could be completed by modifying the dose reduction and treatment schedule. Patients with a low creatinine clearance level at pretreatment and those starting the treatment after total gastrectomy may require careful observation for adverse events, particularly during the early period after treatment.

In the present study, patients with a low creatinine clearance level showed a trend to require dose reduction. Because CDHP, a biochemical modulator of 5-FU, is excreted mainly in the urine, renal function is critical for plasma CDHP clearance. Lower CDHP clearance leads to a prolonged high plasma CDHP concentration, which causes a sustained high plasma 5-FU concentration. This may lead to severe adverse events with an S-1 chemotherapeutic regimen for patients with a low creatinine clearance level. Post-marketing surveillance of $\mathrm{S}-1$ in patients with advanced gastric cancer has demonstrated a close relationship between the incidence of grade 3 or worse hematological toxicities and renal function [11].

With regard to S-1 adverse events, gastrointestinal toxicity has become well recognized, particularly diarrhea, which was the identified dose-limiting toxicity of S-1 in phase I studies in Western countries [12-15]. In the adjuvant setting, these adverse events must be successfully managed to achieve the planned 1-year treatment with S-1. Of the 26 patients who discontinued treatment in the present study, $20(77 \%)$ discontinued due to S-1 adverse events. Persistent gastrointestinal toxicities, even if the grade of adverse events was mild, were the major reasons for patients' refusal to undergo continuous treatment. Therefore, appropriate guidelines must be established for the proper management of adjuvant S-1 therapy in order that the planned 1-year treatment is completed. We recommended to the patients that they skip S-1 administration if they complained of uncomfortable gastrointestinal toxicities. Likewise, we explained to the patients to start taking S-1 again after the relief of symptoms.

Recently, S-1 pharmacokinetic data have demonstrated that the plasma Cmax and AUC of 5-FU after total gastrectomy were significantly higher with S-1 treatment than 
these parameters were before surgery [16-18]. Moreover, statistically significant relationships were observed between the grade of S-1-induced diarrhea and AUC, as well as between the plasma Cmax and 5-FU concentration $[12,15]$. Therefore, it is possible that $\mathrm{S}-1$ toxicities might be enhanced by gastrectomy. In fact, the present study demonstrated that patients who had undergone total gastrectomy had a higher incidence of dose reduction than patients who had subtotal gastrectomy.

Post-hoc analyses of the ACTS-GC trial showed that patients who completed the planned 1-year S-1 treatment had a longer survival than patients who discontinued treatment [19]. Therefore, it is important to complete the planned 1-year treatment, if necessary by modifying the dose or treatment schedule. An appropriate strategy must be developed to achieve completion of treatment and appropriate management of adverse events. As an example, in our patients, when they could take the full 2-week S-1 treatment from the initial treatment but experienced uncomfortable gastrointestinal toxicities that prevented continuation of S-1 administration, we usually changed the treatment schedule from the original 4-week administration followed by a 2-week rest to 2-week administration followed by a 1-week rest. This change in the treatment schedule allows patients to have an earlier rest, which enables them to recover from the prolonged gastrointestinal toxicities or to discontinue treatment before symptoms develop. On the other hand, when patients had not taken the planned S-1 dosage at the time of examination 2 weeks from the initial treatment and had uncomfortable gastrointestinal toxicities that prevented continuation of S-1 administration, one dose-reduction level could be applied as follows: from 120 to $100 \mathrm{mg} /$ day, from 100 to $80 \mathrm{mg}$ / day, or from 80 to $60 \mathrm{mg} / \mathrm{day}$. Thus, the greatest possible efforts to maintain dose intensity must be made.

As for the patients with dose reduction, there was a high probability of requiring dose reduction during the initial 3 months of the planned 1-year treatment. It is therefore deemed necessary that accurate examination and careful treatment must be carried out in light of the adverse events of S-1, particularly until 3 months after the initial S-1 treatment. Significant predictive factors of dose reduction were sought in the present study and in past studies of adjuvant S-1 treatment. We believe that clarification of the predictive factors of S-1 adverse events remains a major issue, because S-1 has been approved for the treatment of several cancers. In further large prospective trials, the exploration of predictive factors of adverse events, such as gene polymorphisms, is expected.

Acknowledgments We thank Eri Oonishi for her invaluable assistance in the preparation of this manuscript. We did not receive any grant support for this study.

\section{References}

1. Kelley JR, Duggan JM. Gastric cancer epidemiology and risk factors. J Clin Epidemiol. 2003;56:1-9.

2. Macdonald JS, Smalley SR, Benedetti J, Hundahl SA, Estes NC, Stemmermann GN, et al. Chemoradiotherapy after surgery compared with surgery alone for adenocarcinoma of the stomach or gastroesophageal junction. N Engl J Med. 2001;345:725-30.

3. Cunningham D, Allum WH, Stenning SP, Thompson JN, Van de Velde CJ, Nicolson M, et al. Perioperative chemotherapy versus surgery alone for resectable gastroesophageal cancer. N Engl J Med. 2006;355:11-20.

4. Sasako M, Sano T, Yamamoto S, Kurokawa Y, Nashimoto A, Kurita A, et al. D2 lymphadenectomy alone or with para-aortic nodal dissection for gastric cancer. N Engl J Med. 2008; 359:453-62.

5. Kinoshita T, Nashimoto A, Yamamura Y, Okamura T, Sasako M, Sakamoto J, et al. Feasibility study of adjuvant chemotherapy with S-1 (TS-1; tegafur, gimeracil, oteracil potassium) for gastric cancer. Gastric Cancer. 2004;7:104-9.

6. Nakajima T, Kinoshita T, Nashimoto A, Sairenji M, Yamaguchi T, Sakamoto J, et al. Randomized controlled trial of adjuvant uracil-tegafur versus surgery alone for serosa-negative, locally advanced gastric cancer. Br J Surg. 2007;94:1468-76.

7. Kato T, Shimamoto Y, Uchida J, Ohshimo H, Abe M, Shirasaka $\mathrm{T}$, et al. Possible regulation of 5-fluorouracil-induced neuro- and oral toxicities by two biochemical modulators consisting of S-1, a new oral formulation of 5-fluorouracil. Anticancer Res. 2001;21: 1705-12.

8. Yamada Y, Hamaguchi T, Goto M, Muro K, Matsumura Y, Shimada $\mathrm{Y}$, et al. Plasma concentrations of 5-fluorouracil and F-beta-alanine following oral administration of S-1, a dihydropyrimidine dehydrogenase inhibitory fluoropyrimidine, as compared with protracted venous infusion of 5-fluorouracil. $\mathrm{Br} \mathrm{J}$ Cancer. 2003;89:816-20.

9. Sakuramoto S, Sasako M, Yamaguchi T, Kinoshita T, Fujii M, Nashimoto A, et al. Adjuvant chemotherapy for gastric cancer with S-1, an oral fluoropyrimidine. N Engl J Med. 2007;357:1810-20.

10. Japanese Gastric Cancer Association. Japanese classification of gastric carcinoma-2nd English edition. Gastric Cancer. 1998;1: $10-24$.

11. Yamanaka T, Matsumoto S, Teramukai S, Ishiwata R, Nagai Y, Fukushima M. Safety evaluation of oral fluoropyrimidine S-1 for short- and long-term delivery in advanced gastric cancer: analysis of 3758 patients. Cancer Chemother Pharmacol. 2008;61:335-43.

12. van Groeningen CJ, Peters GJ, Schornagel JH, Gall H, Noordhuis $\mathrm{P}$, de Vries MJ, et al. Phase I clinical and pharmacokinetic study of oral S-1 in patients with advanced solid tumors. J Clin Oncol. 2000;18:2772-9.

13. Hoff PM, Saad ED, Ajani JA, Lassere Y, Wenske C, Medgyesy $D$, et al. Phase I study with pharmacokinetics of S-1 on an oral daily schedule for 28 days in patients with solid tumors. Clin Cancer Res. 2003;9:134-42.

14. Chu QS, Hammond LA, Schwartz G, Ochoa L, Rha SY, Denis L, et al. Phase I and pharmacokinetic study of the oral fluoropyrimidine S-1 on a once-daily-for-28-day schedule in patients with advanced malignancies. Clin Cancer Res. 2004;10:4913-21.

15. Cohen SJ, Leichman CG, Yeslow G, Beard M, Proefrock A, Roedig B, et al. Phase I and pharmacokinetic study of once daily oral administration of S-1 in patients with advanced cancer. Clin Cancer Res. 2002;8:2116-22.

16. Kochi M, Fujii M, Kanamori N, Kaiga T, Aizaki K, Takahashi T, et al. Effect of gastrectomy on the pharmacokinetics of S-1, an oral fluoropyrimidine, in resectable gastric cancer patients. Cancer Chemother Pharmacol. 2007;60:693-701. 
17. Kim WY, Nakata B, Hirakawa K. Alternative pharmacokinetics of S-1 components, 5-fluorouracil, dihydrofluorouracil and alphafluoro-beta-alanine after oral administration of S-1 following total gastrectomy. Cancer Sci. 2007;98:1604-8.

18. Tsuruoka Y, Kamano T, Kitajima M, Kawai K, Watabe S, Ochiai $\mathrm{T}$, et al. Effect of gastrectomy on the pharmacokinetics of 5-fluorouracil and gimeracil after oral administration of S-1. Anticancer Drugs. 2006;17:393-9.

19. Sakuramoto S, Kikuchi S, Watanabe M. Efficacy of S-1 (oral fluoropyrimidine) for gastric cancer. The Medical Frontline (Saishin Igaku). 2009;64:1075-80. (in Japanese). 